\title{
Influence des hormones androgènes sur le parasitisme des souris mâles par Hymenolepis nana
}

\author{
I. Effets de l'orchiectomie
}

\section{par J. BAILENGER et M.-F. LARCHER-FOURRIER}

Laboratoire de Zoologie et Biologie animale, U.E.R. de Pharmacie, Université de Bordeaux II, F 33000 Bordeaux

\begin{abstract}
Résumé
La castration réduit l'intensité de l'infestation des souris mâles par Hymenolepis nana. Cette diminution de la réceptivité est peu marquée chez les impubères, mais elle est très importante pour les adultes.

Ces résultats sont obtenus avec des rongeurs maintenus dans des conditions excluant toute infestation non contrôlée pour éviter l'interférence des réactions immunologiques.
\end{abstract}

\section{Summary}

The importance of experimental male mice's infestation with Hymenolepis nana is decreased by the orchiectomy. This diminution of their receptivity is light for immature but it is very important for adults.

These results are acquired with mice no infested by helminths during several generations and which are escaped from not controlled contamination during the whole experimentation to avoid the interference of immunological reactions. 
L'intervention des hormones génitales dans la réceptivité parasitaire est envisagée par de nombreux auteurs pour des parasites et des hôtes très différents, mais avec des résultats variables. Le présent travail est limité aux hormones androgènes et au sexe mâle avec, pour hôte, la souris et pour parasite, Hymenolepis nana, Cestode ayant la particularité d'évoluer dans le même organisme, d'une part à l'état larvaire dans la muqueuse intestinale, d'autre part en adulte dans la cavité intestinale tout en conservant néanmoins une fixation sur la muqueuse iléale, au sein des replis poussés par les villosités.

Pour essayer de dégager le rôle des hormones masculinisantes, nous allons étudier les conséquences de l'orchiectomie sur l'évolution du taenia nain, d'une part chez les souris adultes et d'autre part chez les souris impubères maintenues dans des conditions excluant toute infestation non contrôlée afin d'éviter l'interférence des réactions immunologiques.

\section{Méthodes.}

$1^{\circ}$ Gonadectomie : tous les animaux sont castrés à l'âge de trois semaines afin que l'organisme n'ait pratiquement pas été en contact avec les hormones testiculaires et qu'il n'ait donc pas subi les remaniements qu'elles induisent.

Les souris étant très jeunes au moment de l'intervention, on opère par voie abdominale en pratiquant une incision médioventrale, cutanée puis musculaire, d'un centimètre de long à partir du pénis en remontant vers l'avant. L'animal est anesthésié à l'éther ou au Nembutal $(40 \mathrm{mg} / \mathrm{kg})$ selon les expériences et son abdomen rasé puis désinfecté à l'alcool. Les testicules sont enlevés après ligature de leur pédicule vasculaire. Le plan musculaire est refermé par un surjet puis on pulvérise un peu d'un sulfamide avant de recoudre, point par point, le tissu cutané.

En attendant d'infester les souriceaux castrés, on leur applique une prophylaxie antiparasitaire rigoureuse.

$2^{\circ}$ Prophylaxie antiparasitaire : toutes les précautions prises ont pour seul but d'éviter une infestation non contrôlée afin d'éliminer les interférences relevant de l'immunité.

Dès l'arrivée des animaux au Laboratoire, les fèces de chacun d'eux sont analysées et quelques individus de chaque lot sont sacrifiés puis autopsiés pour déceler l'éventuelle présence de Cestodes intestinaux qui conduirait à l'exclusion du lot correspondant.

Les rongeurs sont brossés individuellement avec une poudre au gammexane afin de détruire éventuellement les puces qui pourraient intervenir comme hôte intermédiaire d'H. nana. On les maintient dans une animalerie désinsectisée et aseptisée en les nourrissant avec une alimentation stérilisée et en leur administrant, par tubage gastrique, $250 \mathrm{mg} / \mathrm{kg}$ de Niclosamide finement pulvérisée et en suspension dans de l'eau sucrée, à raison d'une dose hebdomadaire pendant toute la période qui précède l'infestation. Un laps de temps de six jours sépare le dernier traitement de l'infestation. 
Pour s'assurer de leur efficacité, toutes ces mesures prophylactiques sont complétées par un examen coprologique hebdomadaire.

$3^{\circ}$ Infestation : les vers prélevés sur des souris sont laissés au contact d'une solution de chlorure de sodium à 6 p. 1000 pendant 24 heures à la température du Laboratoire. Après ce laps de temps, les strobiles sont éliminés et les œufs, libérés par lyse spontanée des segments mûrs, sont mis en suspension puis numérés dans une cellule de Nageotte. Seuls sont comptés les œufs contenant un embryon hexacanthe. On fait en sorte que la suspension renferme entre 400 et 500 œufs dans un volume de $0,5 \mathrm{ml}$ qui est administré par tubage gastrique.

$4^{\circ}$ Evaluation du parasitisme : 15 jours après l'infestation les souris sont autopsiées, la portion terminale du jejunoiléon est prélevée, fendue longitudinalement, puis les Hymenolepis y sont recherchés et dénombrés. On peut ainsi calculer les pourcentages de souris parasitées ainsi que le nombre moyen de vers qu'elles hébergent et comparer avec des lots témoins.

$5^{\circ}$ Témoins : à chaque lot de souris castrées correspond un lot non gonadectomisé en tous points identique à lui tant en ce qui concerne les animaux, leur conditionnement et leur infestation.

\section{Résultats}

L'influence de la castration est étudiée, d'une part sur les impubères et d'autre part sur les adultes.

Tableau I.

Influence de l'orchiectomie par le parasitisme des souris par Hymenolepis nana

\begin{tabular}{|c|c|c|c|c|c|c|c|c|c|c|}
\hline \multirow{2}{*}{$\begin{array}{c}\text { Ages } \\
\text { (semai- } \\
\text { nes) }\end{array}$} & \multicolumn{4}{|c|}{ Animaux non castrés } & \multicolumn{4}{|c|}{ Animaux castrés } & \multicolumn{2}{|c|}{ Rapports *nt } \\
\hline & Nbre & $\%$ & infesté & $\begin{array}{c}\text { Nbre } \\
\text { de } \\
\text { vers * }\end{array}$ & Nbre & $\%$ & infesté & $\begin{array}{c}\text { Nbre } \\
\text { de } \\
\text { vers * }\end{array}$ & \% infesté & $\begin{array}{c}\text { Nbre } \\
\text { de } \\
\text { vers * }\end{array}$ \\
\hline $5 \ldots$ & 12 & & 75 & 1,7 & 13 & & 53 & 1,1 & 1,4 & 1,5 \\
\hline ... & 13 & & 100 & 6,8 & 14 & & 14 & 1,5 & 7 & 4,5 \\
\hline $14 \ldots$ & 13 & & 76 & 4,6 & 14 & & 7 & 1 & 10,7 & 4,6 \\
\hline
\end{tabular}

* Nombre de vers : il s'agit du nombre moyen par souris parasitée.

w.* Rapports : $\frac{\text { Témoins }}{\text { Castrats }}$ 
$1^{\circ}$ Souris impubères : l'infestation de 25 animaux de 5 semaines répartis en 2 lots - 12 non castrés et 13 castrés à l'âge de 3 semaines - conduit à des pourcentages de parasitisme qui atteignent respectivement 75 et 53 p. 100 avec des moyennes de 1,7 et 1,1 vers par souris parasitée (tableau I). La castration semble diminuer la réceptivité des souris mâles de 5 semaines.

$2^{\circ}$ Souris adultes : on réalise deux séries expérimentales de deux lots composés de 12 à 14 animaux chacun et portant respectivement sur des rongeurs âgés, lors de l'infestation, de 9 et 14 semaines.

Les résultats portés au tableau I sont particulièrement nets : les souris mâles castrées sont nettement moins parasitées que les rongeurs entiers. On aboutit à cette conclusion en considérant aussi bien les pourcentages d'animaux infestés que le nombre moyen de vers qu'ils portent: les souris de 9 semaines non castrées sont toutes parasitées avec une moyenne de 6,8 vers, tandis que pour les souris castrées de même âge, ces valeurs sont respectivement de $14 \%$ et 1,5 vers; à 14 semaines, $76 \%$ des animaux non castrés se parasitent avec une moyenne de 4,6 vers, mais après castration le pourcentage tombe à 7 et la moyenne à 1 .

\section{Discussion}

Les résultats obtenus apportent la preuve indiscutable que l'ablation des testicules diminue la réceptivité des souris à l'égard d'Hymenolepis nana. Cette même notion se dégage d'un certain nombre d'autres travaux portant sur des vers parasites différents: Nippostrongylus brasiliensis (Solomon 6, 7), Aspiculuris tetraptera (Mathies 5), Schistosoma mansoni (Berg 4), Hymenolepis diminuta (Beck 3, Addis 1), Echinococcus granulosus (8).

Dans notre expérimentation, ce phénomène paraît être d'autant plus net que les rongeurs sont plus âgés.

Pour les lots non castrés et castrés d'un même âge qui ont été conditionnés et infestés avec une similitude totale, il est possible d'établir les rapports d'une part entre les pourcentages des animaux parasités et, d'autre part, entre les nombres moyens de vers qu'ils hébergent. On observe que ces rapports augmentent avec l'âge : à 5 semaines, les animaux témoins sont 1,4 fois plus parasités que les castrats et le rapport entre les nombres de vers est égal à 1,5; à 9 semaines, les souris non castrées sont 7 fois plus fréquemment parasitées que les castrats et ceci avec un nombre de vers 4,5 fois supérieur ; ces valeurs sont respectivement de 10,7 et 4,6 à 14 semaines.

Nous avons, par ailleurs (2), démontré l'absence d'influence de l'âge sur l'atteinte parasitaire, laquelle est sensiblement constante entre 4 et 14 semaines. La castration modifie donc un équilibre anatomo-histo-physiologique dans le sens d'une réceptivité amoindrie. La nature de cette modification reste à préciser. 


\section{Conclusion}

L'orchiectomie entraine une diminution de l'infestation des souris par Hymenolepis nana. Cet amoindrissement de la réceptivité est d'autant plus marqué que les animaux sont plus âgés : faible à 5 semaines, il devient particulièrement important chez des rongeurs de 9 et 14 semaines. L'expérimentation est faite avec des rongeurs maintenus dans des conditions excluant toute infestation non contrôlée afin d'éviter l'interférence des réactions immunologiques.

\section{Bibliographie}

1. Addis (C. J.), 1946. - Experiments on relation between sex hormones and the growth of tapeworm $H$. diminuta in rats. $J$. of Parasitology, 32, 574-580.

2. Bailenger (J.), Faragit (G.), Larcher-Fourrier (M.-F.) et Cerbelle (F.), 1972. Influence de l'âge et du sexe sur le parasitisme des souris par Hymenolepis nana. Ann. Paras. Hum. comp., 47, 767-772.

3. BECK (J.W.), 1952. - Effect of gonadectomy and gonadal hormones on singly established $H$. diminuta in rats. Exp. parasitology, 1, 109-117.

4. Berg (E), 1953. - Effect of castration in mâle mice on Schistosoma mansoni : Proceeding of the society for experimental biology and medecine, 83-85.

5. Mathies (A. W.), 1954. - Effects of sex on mouse pinworm infections. J. of parasitology, 40,702 .

6. Solomon (G. B.), 1963. - Effects of gonadectomy and hormone therapy on Nippostrongylus brasiliensis. J. of parasitology, 48, supp. 37.

7. - 1966. - Developpement of Nippostrongylus brasiliensis in gonadectomised and hormone treated Hamsters. Experimental parasitology, 18, 374-396.

8. Szidat (L.), 1971. - Neue aspekte des Echinococcen-Problems. Angew Parasit., 12, 133143. 